\title{
Changes in diad sequence distribution by preferential chain scission during the thermal hydrolysis of poly(3-hydroxybutyrate-co-3-hydroxyhexanoate)
}

\author{
Dhurga Devi Rajaratanam ${ }^{1,2}$, Hidayah Ariffin ${ }^{1,3}$, Mohd Ali Hassan ${ }^{1}$ and Haruo Nishida ${ }^{2}$
}

Polymer Journal (2016) 48, 839-842; doi:10.1038/pj.2016.28; published online 16 March 2016

\section{INTRODUCTION}

Polyhydroxyalkanoates (PHAs) are microbial polyesters produced by many types of bacteria as an intracellular energy reserve material under substrate limiting conditions and in the presence of excessive carbon sources. ${ }^{1}$ Poly $((R)-3$-hydroxybutyrate) (PHB), the most commonly used microbial polyester, was the first member of the PHA family to be discovered, and more than 150 other monomer units have been reported to date. ${ }^{2,3} \operatorname{Poly}((R)$-3-hydroxybutyrate-co$(R)$-3-hydroxyhexanoate) (PHBHHx) is a copolymer in the PHA family that consists of randomly distributed $(R)$-3-hydroxybutyrate (HB) and (R)-3-hydroxyhexanoate (HHx) units. ${ }^{4}$ This type of copolymer exhibits improved mechanical properties and processability compared with those of $\mathrm{PHB}$ and poly $((R)$-3-hydroxyvalerate) (PHBV). ${ }^{5}$ PHBHHx copolymers are currently produced on a large scale and have proven to be biocompatible in clinical studies using mouse fibroblasts cells, and rabbit articular cartilage-derived chondrocytes. ${ }^{6}$ PHBHHx is a highly favorable copolymer of the PHB family due to its biodegradability, flexible mechanical properties and good melt processability.

Because PHBHHx has potential applications in various fields, the degradation properties of PHBHHx must be determined, especially those related to thermal degradation and hydrolysis, which causes substantial changes in the mechanical properties after melt-processing. Previously, degradation studies on members of the PHA family have mainly focused on enzymatic degradation, ${ }^{7,8}$ thermal degradation ${ }^{9-10}$ and hydrothermal degradation, ${ }^{11}$ as well as acid and alkaline hydrolysis. ${ }^{12}$ The resulting degradation products are dependent on the degradation mechanisms. The thermal degradation of PHB occurs through random chain scissions via $\beta$-elimination, resulting in final products with an unsaturated chain end. In the hydrothermal degradation of $\mathrm{PHB}$, homogeneous and random degradation occur via bulk erosion at high temperature $\left(180-300^{\circ} \mathrm{C}\right)$ and high pressure, which result in the formation of water-soluble monomers and oligomers. $^{11}$

Previously, studies on the hydrolysis of $\mathrm{PHBHHx}$ have shown that hydrolysis occurs preferentially at the amorphous phase, rather than the crystalline phase. ${ }^{13-15}$ However, the effects of $\mathrm{HHx}$ units in the $3 \mathrm{HB}$ unit sequence on the hydrothermal degradation of $\mathrm{PHBHHx}$ have not yet been determined. To evaluate the effect of HHx units on hydrothermal degradation, super-heated steam (SHS) hydrolysis was introduced as an alternative method for the hydrolysis of PHBHHx. SHS uses unsaturated steam produced by the addition of heat to saturated steam, which enables the steam's temperature to exceed its boiling point. ${ }^{16}$ SHS is widely applicable because it is operable at atmospheric pressure, ${ }^{17}$ allows steam to homogeneously diffuse into PHBHHx, and specifically degrades ester bonds without any dissolving out of hydrolysates into a liquid solvent, which interrupts quantitative analysis of degradation behavior. ${ }^{18}$

In the present study, the effects of HHx units on the hydrothermal degradation of $\mathrm{PHBHHx}$ with $6 \% \mathrm{HHx}$ were investigated and compared with that of $\mathrm{PHB}$, with a focus on changes in the diad sequence distribution after SHS hydrolysis. The diad sequence distribution for $\mathrm{PHBHHx}$ has been determined in previous studies; ${ }^{13-14}$ however, to date changes in diad sequence distribution during degradation, that is, the effects of $\mathrm{HHx}$ on the sequence during hydrolysis, remain unknown. To clarify these effects, changes in the first-order structure of PHBHHx during SHS hydrolysis were monitored by precise structural analyses, including size-exclusion chromatography (SEC), and proton $\left({ }^{1} \mathrm{H}\right)$ and carbon $\left({ }^{13} \mathrm{C}\right)$ nuclear magnetic resonance (NMR) spectroscopies. Moreover, the reaction mechanism was validated by performing a lowestunoccupied molecular orbital (LUMO) analysis on model compounds.

\footnotetext{
${ }^{1}$ Department of Bioprocess Technology, Faculty of Biotechnology and Biomolecular Sciences, Universiti Putra Malaysia, Serdang, Malaysia; ${ }^{2}$ Department of Biological Functions Engineering, Graduate School of Life Science and Systems Engineering, Kyushu Institute of Technology, Fukuoka, Japan and ${ }^{3}$ Laboratory of Biopolymer and Derivatives, Institute of Tropical Forestry and Forest Product (INTROP), Universiti Putra Malaysia, Serdang, Malaysia

Correspondence: Dr H Ariffin, Department of Bioprocess Technology, Faculty of Biotechnology and Biomolecular Sciences, Laboratory of Biopolymer and Derivatives, Institute of Tropical Forestry and Forest Product (INTROP), Universiti Putra Malaysia, 43400 Serdang, Malaysia.

or Professor H Nishida, Graduate School of Life Science and Systems Engineering, Kyushu Institute of Technology, 2-4 Hibikino, Wakamatsu, Kitakyushu, Fukuoka 808-0196, Japan. E-mail: hidayah@upm.edu.my or nishida@lsse.kyutech.ac.jp

Received 24 September 2015; revised 25 December 2015; accepted 13 January 2016; published online 16 March 2016
} 


\section{EXPERIMENTAL PROCEDURE}

Characterization details: $\mathrm{PHBHHx}$ with $6.0 \% \mathrm{HHx}$, $(\operatorname{poly}(R)$-3-hydroxybutyrate-co-6\%-(R)-3-hydroxyhexanoate) and PHB were subjected to the purification process described in the Supplementary Information. The films were prepared as follows: approximately $5 \mathrm{~g}$ of PHA was weighed and placed on top of a metal mold $\left(10 \times 10 \mathrm{~cm}^{2}, 0.5\right.$-mm thickness $)$ with a teflon-coated bottom. The sample was positioned by an up-down close-up with $\mathrm{Al}$ plates. The sample was preheated on a hotplate at 145 and $170{ }^{\circ} \mathrm{C}$ for PHBHHx and PHB samples, respectively, before being pressed at $10 \mathrm{MPa}$ for $3 \mathrm{~min}$, and then at $40-50 \mathrm{MPa}$ for $5 \mathrm{~min}$ to form transparent films. The films were cut into sample strips $\left(1 \times 4 \mathrm{~cm}^{2}, 0.5-\mathrm{mm}\right.$ thickness), which were evaluated in the SHS treatment. The sample strips were treated under the following conditions: 200-600 $\mathrm{min}$ at regular intervals of 20,30, 40 and $60 \mathrm{~min}$, and temperatures of 190, 170, 150 and $130^{\circ} \mathrm{C}$, respectively. After SHS treatment, the samples were placed in a desiccator at room temperature overnight until constant weights were obtained. Subsequently, the sample strips were kept in an airtight bag at $4{ }^{\circ} \mathrm{C}$ before analysis.

A detailed description of the sample characterization procedure is also provided in the Supplementary Information. Molecular weight changes were analyzed with SEC. Proton $\left({ }^{1} \mathrm{H}\right)\left(500 \mathrm{MHz}, \mathrm{CDCl}_{3}\right)$ and carbon $\left({ }^{13} \mathrm{C}\right) \mathrm{NMR}$ $\left(125 \mathrm{MHz}, \mathrm{CDCl}_{3}\right)$ spectroscopies were applied to determine the composition and diad sequence distribution of HB and HHx subunits. Semi-empirical molecular orbital calculations were performed on a TOSHIBA Dynabook N51/25M (TOSHIBA, Tokyo, Japan), equipped with a $2.16 \mathrm{GHz}$ Intel Celeron CPU N28 processor using Hulinks Spartan '08 software (Hulinks, Tokyo, Japan). The equilibrium geometries were optimized using the Hartree-Fock/3$21 \mathrm{G}^{*}$ method to obtain the LUMO map, which displayed the absolute value of the LUMO on the electron density surface of each model compound.

\section{RESULTS AND DISCUSSION}

The structural and thermal properties of PHBHHx containing 6.0\% $\mathrm{HHx}$ unit content as well as PHB, which was used as a reference, are shown in Supplementary Table SI-1 (Supplementary Information). According to the characterization results, PHBHHx possessed two endothermic peaks at 128 and $143^{\circ} \mathrm{C}$, and a low crystallinity value of $26 \%$, which was calculated from the whole sample by differential scanning calorimetry analysis. In contrast, PHB showed a melting point and crystallinity value of $170{ }^{\circ} \mathrm{C}$ and $50 \%$, respectively. The SHS hydrolysis of PHBHHx and PHB was carried out at 130, 150, 170 and $190^{\circ} \mathrm{C}$. At these temperatures, PHBHHx was practically in a molten state, whereas PHB maintained its crystalline structure at 130 and $150^{\circ} \mathrm{C}$. Changes in the SEC profiles of SHS-treated PHBHHx and PHB are illustrated in Supplementary Figures SI-1-8 (Supplementary Information). The SEC profile shifted to a lower molecular weight region but maintained a unimodal distribution profile, similar to the most probable distribution, up to a critical point. The most probable distribution indicates that hydrothermal degradation due to SHS treatment proceeded in a homogeneous, random degradation manner via bulk erosion mechanism. ${ }^{18,19}$ Beyond the critical point, the SEC profile gradually changed to a multimodal distribution, indicating that degradation became heterogeneous and random. However, this result was different from those previously obtained when hydrolysis was performed at high temperatures $\left(180-300{ }^{\circ} \mathrm{C}\right)$ and high pressures. ${ }^{11}$ The observed behavior was similar to the manner accompanying the isolation of crystalline phases during poly(L-lactic acid) hydrolysis ${ }^{18}$. At 170 and $190^{\circ} \mathrm{C}$, in spite of the molten state, the critical point was clearly observed at $M_{\mathrm{w}:} 32500(150 \mathrm{~min})$ and $20000 \mathrm{Da}(80 \mathrm{~min})$, respectively, for PHB, and at $M_{\mathrm{w}}: 29000(180 \mathrm{~min})$ and $23500 \mathrm{Da}$ (80 min), respectively, for PHBHHx. Thus, these suggest that either the dominant and homogeneous bulk erosion mechanism reached the limit or the chain ends were heterogeneously distributed around the original crystalline phases due to the placement of samples in a static state at atmospheric pressure without agitation during SHS treatment.
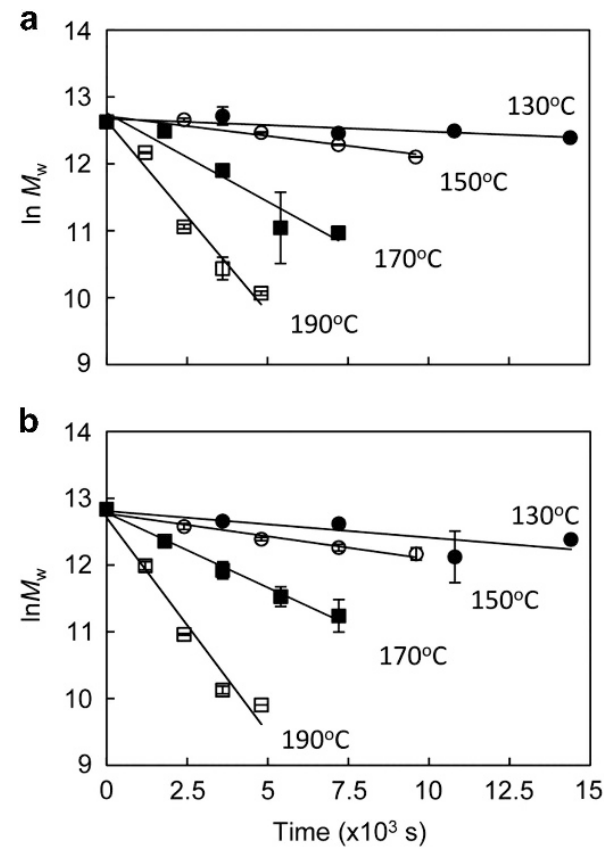

Figure 1 Plots of In $M_{\mathrm{w}}$ versus time for (a) PHBHHx and (b) PHB during SHS treatment, which was performed at temperatures of $130-190^{\circ} \mathrm{C}$. The bars represent the s.d.

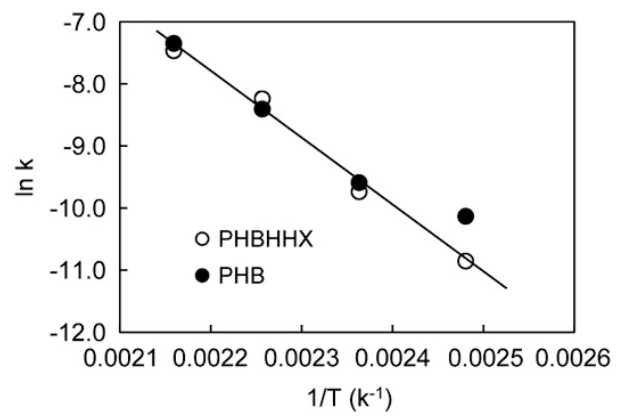

Figure 2 Arrhenius plots for the SHS degradation of PHBHHx and PHB.

The relationship between $\ln M_{\mathrm{w}}$ and treatment time of PHBHHx and PHB is shown in Figures 1a and b, respectively. In the plot, $M_{\mathrm{w}}$ was adopted to calculate the kinetic parameters, to improve the precision of the calculation, as previously reported. ${ }^{18}$ The reaction time and $\ln M_{\mathrm{w}}$ presented a linear relationship, indicating that the reaction proceeded according to autocatalytic random degradation kinetics. ${ }^{20}$ The reaction rate constant $k$ was calculated with Nishida's method, as shown in Supplementary Table SI-2, and the Arrhenius plots were obtained using $\ln k$ and $1 / T$, as depicted in Figure 2. According to Figure 2, a strong linear relationship was obtained for PHBHHx, resulting in an activation energy value of $\left(E_{\mathrm{a}}\right)$ $90.6 \mathrm{~kJ} \bullet \mathrm{mol}^{-1}$, whereas the plot of PHB deviated from linearity at $130^{\circ} \mathrm{C}$, providing a higher $k$ value due to the accelerating effect of concentrated chain-end groups around crystalline phases, which acted as catalysts and hydrophilic groups. At temperatures of 170 and $190^{\circ}$ $\mathrm{C}$, both samples were present in the molten state; therefore, their $k$ values were similar. The tentative $E_{\mathrm{a}}$ of PHB hydrolysis at temperatures of $150-190^{\circ} \mathrm{C}$ was estimated to be $91.4 \mathrm{~kJ} \bullet \mathrm{mol}^{-1}$, which was similar to the $E_{\mathrm{a}}$ of PHBHHx hydrolysis, reflecting the dominance of $\mathrm{HB}-\mathrm{HB}$ bond cleavage. 


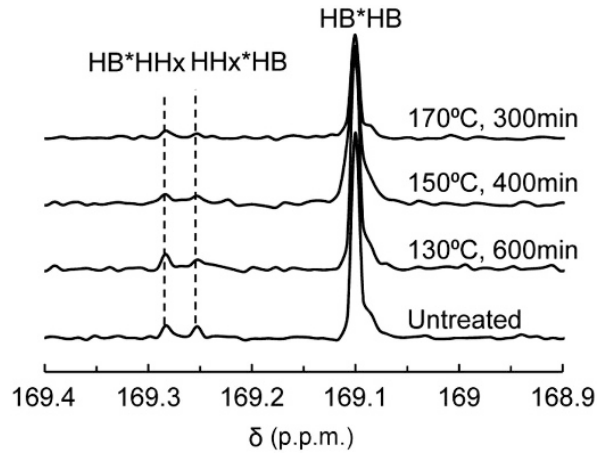

Figure 3 The carbonyl resonances of diad sequences in the ${ }^{13} \mathrm{C} N M R$ spectra of PHBHHx after SHS treatment under different conditions.

The significantly higher value of $\mathrm{PHB}$ at $130^{\circ} \mathrm{C}$, the temperature at which the crystalline phase of PHB remained intact, was due to the limited area of the amorphous phase, in which carboxyl end groups were concentrated. Concentrated carboxyl chain-end groups probably catalyzed the hydrolysis of surrounding ester groups, resulting in a higher rate of hydrolysis.

To elucidate the effects of HHx on the SHS degradation of PHBHHx, changes in the diad sequence distribution were calculated on the basis of the integration values of the carbonyl resonance peaks in the ${ }^{13} \mathrm{C} N M R$ spectra for the following diad sequences: $\mathrm{HB}^{\star} \mathrm{HB}, \mathrm{HB}^{\star} \mathrm{HHx}$ and $\mathrm{HHx}^{\star} \mathrm{HB}$ at $169.100,169.253$ and 169.284 p.p.m., respectively (Figure 3). Each resonance peak was simulated using the Lorentzian function to estimate the peak area, as shown in Supplemetary Table SI-3. The calculated diad sequence distribution values of PHBHHx were compared with values calculated with Bernoullian statistics, which are applicable to statistically random copolymerizations.

In the Bernoullian model, the mole fraction $F_{i^{*} j}$ of diad sequence $i^{*} j$ can be expressed as $F_{i * j}=F_{i} F_{j}$. The mole fractions $F_{i}$ and $F_{j}$ have units of $i$ and $j$, respectively. The calculated diad fractions: $F_{\mathrm{HB}^{*} \mathrm{HB}}, F_{\mathrm{HB}^{*} \mathrm{HHx}}$, $F_{\mathrm{HHx}^{*} \mathrm{HB}}$ and $F_{\mathrm{HHx}^{*} \mathrm{HHx}}$ of original PHBHHx were 88.36, 5.64, 5.64 and $0.36 \%$, respectively, which was in agreement with the observed values: 88.6, 5.8, 5.6 and $\sim 0 \%$ for $F_{\mathrm{HB}^{*} \mathrm{HB}}, F_{\mathrm{HB}^{*} \mathrm{HHx}}, F_{\mathrm{HHx}^{*} \mathrm{HB}}$ and $F_{\mathrm{HHx}{ }^{*} \mathrm{HH}}$, respectively. The peak corresponding to diad sequence fraction $F_{\mathrm{HHx}^{*} \mathrm{HHx}}$ was difficult to detect in the ${ }^{13} \mathrm{C}$ NMR spectrum due to the low amount of $\mathrm{HHx}^{\star} \mathrm{HHx}$ sequences. Thus, the sequence distribution of $\mathrm{HB}$ and $\mathrm{HHx}$ units in $\mathrm{PHBHHx}$ is statistically random. ${ }^{14}$

As shown in Figure 3 and Supplemetary Table SI-3, the relative peak intensity of the diad sequence $\mathrm{HHx}^{\star} \mathrm{HB}$ gradually decreased, whereas the intensities of $\mathrm{HB}^{\star} \mathrm{HB}$ and $\mathrm{HB}^{\star} \mathrm{HHx}$ diad sequences increased considerably. The observed changes in the relative peak intensities of the diad sequences are clearly illustrated in Figure 4. Although untreated $\mathrm{PHBHHx}$ showed similar intensity values of $5.8 \%$ and $5.6 \%$ for $\mathrm{HB}^{\star} \mathrm{HHx}$ and $\mathrm{HHx}^{\star} \mathrm{HB}$ sequences, respectively, after the SHS treatment was performed at $170{ }^{\circ} \mathrm{C}$ for $300 \mathrm{~min}$, the relative intensity of $\mathrm{HHx}^{\star} \mathrm{HB}$ decreased to $3.9 \%$, whereas the value of $\mathrm{HB}^{\star} \mathrm{HHx}$ increased to $6.9 \%$. After treatment at $190{ }^{\circ} \mathrm{C}$ for $200 \mathrm{~min}$, the spectrum broadened because of the extreme decrease in molecular weight (Supplementary Table SI-3), and the relative intensities of $\mathrm{HHx}^{\star} \mathrm{HB}$ and $\mathrm{HB}^{\star} \mathrm{HHx}$ were tentatively simulated using the Lorentzian function, showing calculated values of $3.6 \%$ and $7.1 \%$, respectively. Moreover, the relative intensity of $\mathrm{HB}^{\star} \mathrm{HB}$ also increased slightly from 88.6 to $89.3 \%$. $\mathrm{HHx}^{\star} \mathrm{HB}$ sequences were preferentially degraded, and the chain scission of $\mathrm{HB}^{\star} \mathrm{HHx}$ sequences was suppressed during SHS treatment (Scheme 1). These specific reaction

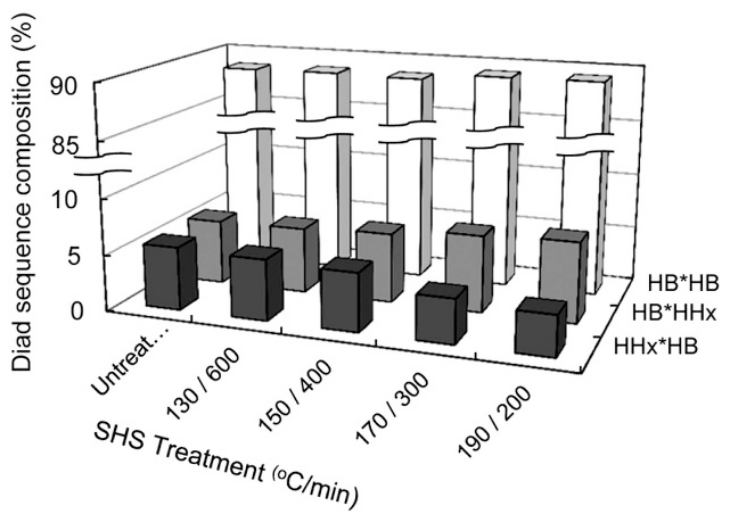

Figure 4 Changes in the diad sequence distribution of SHS-treated $\mathrm{PHBHHx}$.
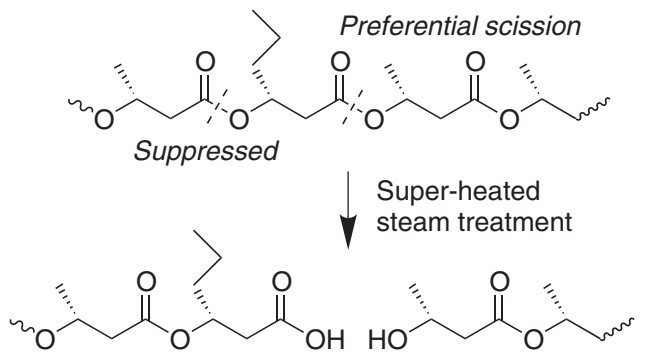

Scheme 1 Schematic depiction of the specific hydrolysis properties of $\mathrm{PHBHHx}$.

properties were attributed to the greater steric effects of the propyl group in the HHx unit compared with the methyl group in the HB unit. Specifically, the propyl group had a promoting effect due to the concentration of HHx units in amorphous regions, as well as a suppressive effect due to the steric hindrance and hydrophobicity of the propyl group.

The key mechanism of ester hydrolysis is nucleophilic acyl substitution, in which the ester carbonyl carbon is the target of the nucleophile. Given that the LUMO designates the space available for a pair of electrons, a LUMO map indicates the most probable location of nucleophilic attack. Three model compounds, including Me-O- $(R)$ $3 \mathrm{HB}-(R)-3 \mathrm{HB}-\mathrm{Me}$, Me-O- $(R)-3 \mathrm{HB}-(R)-3 \mathrm{HHx}-\mathrm{Me}$ and $\mathrm{Me}-\mathrm{O}-(R)-$ $3 \mathrm{HHx}-(R)-3 \mathrm{HB}-\mathrm{Me}$, were applied to calculate the LUMO and estimate the hydrolyzability of ester carbonyl carbons after nucleophilic attack. The LUMO maps for the model compounds, on which the absolute value of the LUMO is depicted on electron density surfaces, are shown in Supplementary Figures SI-9-11 (Supplementary Information). In the figures, the blue color indicates a high concentration of the LUMO, which showed the highest concentration over ester carbonyl carbons. The LUMO values of the carbonyl carbons are listed in Supplementary Table SI-4 (Supplementary Information). The highest absolute LUMO value was observed for the $\mathrm{HHx}^{\star} \mathrm{HB}$ carbonyl carbon $\left(115.08 \mathrm{~kJ} \bullet \mathrm{mol}^{-1}\right)$, followed by $\mathrm{HB}^{\star} \mathrm{HB}\left(112.95 \mathrm{~kJ} \bullet \mathrm{mol}^{-1}\right)$ and $\mathrm{HB}^{\star} \mathrm{HHx}\left(109.58 \mathrm{~kJ}^{\circ} \mathrm{mol}^{-1}\right)$ carbonyl carbons. These results are in agreement with the diad sequence distribution data shown in Figure 4 and Supplementary Table SI-3. On the basis of these results, the $\mathrm{HHx}^{\star} \mathrm{HB}$ carbonyl carbon in $\mathrm{PHBHHx}$ had the highest absolute LUMO value, and acid-catalyzed hydrolysis ${ }^{18}$ was accelerated in $\mathrm{HHx}^{\star} \mathrm{HB}$ sequences during the SHS treatment. 


\section{CONCLUSION}

To clarify the effects of co-monomer units on the hydrothermal degradation of microbial polyesters, the degradation specificity of PHBHHx in hydrolysis was investigated using SHS. The results showed that the degradation of $\mathrm{PHBHHx}$ proceeded via autocatalytic random degradation kinetics, with an activation energy of $E_{\mathrm{a}}=90.6 \mathrm{~kJ} \bullet \mathrm{mol}^{-1}$ at a temperature of $130-190^{\circ} \mathrm{C}$. According to the observed changes in the diad sequence distribution, $\mathrm{HHx}{ }^{\star} \mathrm{HB}$ sequences were preferentially hydrolyzed, whereas the hydrolysis of neighboring $\mathrm{HB}^{\star} \mathrm{HHx}$ sequences was suppressed. This degradation specificity was successfully supported by the LUMO analysis of model compounds. The aforementioned reaction properties were attributed to increased steric effects of the propyl group in the HHx unit. Additional experiments using other copolymers with different HHx contents are currently in progress and will be reported in the near future.

\section{CONFLICT OF INTEREST}

The authors declare no conflict of interest.

\section{ACKNOWLEDGEMENTS}

We would like to extend our deepest gratitude to Kaneka Corporation, Japan, for providing PHBHHx samples and to the Japanese Government for providing scholarships for short-term studies in Japan, which were supported by the Japan Student Services Organization (JASSO). This project was funded by the Fundamental Research Grant Scheme (FRGS, grant no. 5524483) of the Ministry of Higher Education, Malaysia.

1 Tian, P. Y., Shang, L., Ren, H., Mi, Y., Fan, D. D. \& Jiang, M. Biosynthesis of polyhydroxyalkanoates: current research and development. Afr. J. Biotechnol. 8, 709-714 (2009).

2 Keshavarz, T. \& Roy, I. Polyhydroxyalkanoates: bioplastics with a green agenda. Curr. Opin. Microbiol. 13, 321-326 (2010).

3 Chen, G. Q. in Plastics From Bacteria: Natural Functions and Applications (ed. Chen G. Q.) (Springer-Verlag Berlin Heidelberg, London, UK, 2010).

4 Yang, Y., Ke, S., Ren, L., Wang, Y., Li, Y. \& Huang, H. Dielectric spectroscopy of biodegradable poly(3-hydroxybutyrate-co-3-hydroxyhexanoate) films. Eur. Polym. J. 48, 79-85 (2012).
5 Wang, Y. W., Mo, W., Yao, H., Wu, Q., Chen, J. \& Chen, G. Q. Biodegradation studies of poly(3-hydroxybutyrate-co-3-hydroxyhexanoate). Polym. Degrad. Stab 85, 815-821 (2004).

6 Zheng, Z., Bei, F. F., Tian, H. L. \& Chen, G. Q. Effects of crystallization of polyhydroxyalkanoate blend on surface physicochemical properties and interactions with rabbit articular cartilage chondrocytes. Biomaterials 26, 3537-3548 (2005).

7 Sudesh, K., Abe, H. \& Doi, Y. Synthesis, structure and properties of polyhydroxyalkanoates: biological polyesters. Prog. Polym. Sci. 25, 1503-1555 (2000).

8 Alejandra, R., Margarita, C. \& Soledad, M. M. Enzymatic degradation of poly(3hydroxybutyrate) by a commercial lipase. Polym. Degrad. Stab. 97, 2473-2476 (2012).

9 Ariffin, H., Nishida, H., Shirai, Y. \& Hassan, M. A. Highly selective transformation of poly[(R)-3-hydroxybutyric acid] into trans-crotonic acid by catalytic thermal degradation. Polym. Degrad. Stab. 95, 1375-1381 (2010).

10 Ramier, J., Grande, D., Langlois, V. \& Renard, E. Toward the controlled production of oligoesters by microwave-assisted degradation of poly(3-hydroxyalkanoate)s. Polym. Degrad. Stab. 97, 322-328 (2012).

11 Saeki, T., Tsukegi, T., Tsuji, H., Daimon, H. \& Fujie, K. Hydrolytic degradation of poly[(R)-3-hydroxybutyric acid] in the melt. Polymer 46, 2157-2162 (2005).

$12 \mathrm{Yu}$, G. \& Marchessault, R. H. Characterization of low molecular weight poly $(\beta$ hydroxybutyrate)s from alkaline and acidic hydrolysis. Polymer 41, 1087-1098 (2000).

13 Shimamura, E., Kasuya, K., Kobayashi, G., Shiotani, T., Shima, Y. \& Doi, Y. Physical properties and biodegradability of microbial poly(3-hydroxybutyrate-co-3-hydroxyhexanoate). Macromolecules 27, 878-880 (1994).

14 Doi, Y., Kitamura, S. \& Abe, H. Microbial synthesis and characterization of poly(3- hydroxybutyrate-co-3-hydroxyhexanoate). Macromolecules 28, 4822-4828 (1995)

15 Yang, H. X., Sun, M. \& Zhou, P. Investigation of water diffusion in poly(3-hydroxybutyrate-co-3-hydroxyhexanoate) by generalized two-dimensional correlation ATRFTIR spectroscopy. Polymer 50, 1553-1540 (2009).

16 Bahrin, E. K., Baharuddin, A. S., Ibrahim, M. F., Razak, M. N. A., Sulaiman, A., Aziz, S. A., Hassan, M. A., Shirai, Y. \& Nishida, H. Physicochemical property changes and enzymatic hydrolysis enhancement of oil palm empty fruit bunches treated with superheated steam. Bioresources 7, 1784-1801 (2012).

17 Nordin, N. I. A. A., Ariffin, H., Andou, Y., Hassan, M. A., Shirai, Y., Nishida, H., Yunus, W. M. Z. W., Karuppuchamy, S. \& Ibrahim, N. A. Modification of oil palm mesocarp fiber characteristics using superheated steam treatment. Molecules 18, 9132-9146 (2013).

18 Mohd-Adnan, A. F., Nishida, H. \& Shirai, Y. Evaluation of kinetics parameters for poly (L-lactic acid) hydrolysis under high-pressure steam. Polym. Degrad. Stab. 93, 1053-1058 (2008)

19 Tsuji, H., Nakahara, K. \& Ikarashi, K. High-temperature hydrolysis of poly(L-Lactide) films with different crystallinities and crystalline thickness in phosphate-buffered solution. Macromol. Mater. Eng. 286, 398-406 (2001)

20 Nishida, H., Yamashita, M., Nagashima, M., Hattori, N., Endo, T. \& Tokiwa, Y. Theoretical prediction of molecular weight on auto-catalytic random hydrolysis of aliphatic polyesters. Macromolecules 33, 6595-6601 (2000).

Supplementary Information accompanies the paper on Polymer Journal website (http://www.nature.com/pj) 